
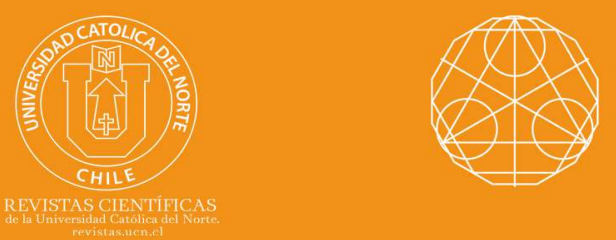

\title{
Bounds on linear codes capable of detecting, locating and correcting of repeated burst errors prevailing in multiple sub-blocks
}

Pankaj Kumar Das ${ }^{1}$ (1) orcid.org/0000-0003-2197-2389

Subodh Kumar ${ }^{2}$ (1) orcid.org/0000-0002-3242-9333

${ }^{1}$ Tezpur University, Dept. of Mathematical Sciences, Sonitpur, AS, India.

pankaj4thapril@yahoo.co.in

${ }^{2}$ University of Delhi, Shyam Lal College, Dept. of Mathematics, Delhi, India.

subodh05031981@gmail.com

\section{Abstract:}

Linear codes are presented that can detect, locate and correct all repeated burst errors of length $b$ or less which occur in multiple sub-blocks. We obtain lower and upper bounds on the number of check digits for the existence of these codes. Three examples, one for each type of code, are provided.

Keywords: Syndromes; Parity check matrix; Bound; Burst; Repeated burst.

MSC (2020): 94B05, 94B65.

\section{Cite this article as (IEEE citation style):}

P. K. Das and S. Kumar, "Bounds on linear codes capable of detecting, locating and correcting of repeated burst errors prevailing in multiple sub-blocks", Proyecciones (Antofagasta, On line), vol. 39, no. 6, pp. 1577-1596, Dec. 2020, doi: 10.22199/issn.07176279-2020-06-0094.

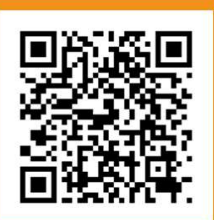

Article copyright: (C) 2020 Pankaj Kumar Das and Subodh Kumar. This is an open access article distributed under the terms of the Creative Commons License, which permits unrestricted use and distribution provided the original author and source are credited. 


\section{Introduction}

In the history of coding theory, earlier the study was revolving around the detection and correction of random and burst errors. If we want to detect the error, relatively shorter length code can do the job. If we want to do error correction, the code length needs to be lengthened, but it results in low data rate of transmission. In order to sort out this problem or to make a compromise between short and long code length, Wolf and Elpas [13] in 1963 gave a new middle technique/concept. In this concept, the whole code length is divided in some mutually exclusive smaller sub-blocks and the errors are assumed to be occurred within sub-block(s). This helps to make a balance in the efficiency of the channel. Many papers are written in this direction, e.g., $[2,3,4,8,12]$.

In paper [2], Das obtains bounds for linear code which can locate burst errors affecting more than one sub-block, whereas the paper [3] presents random error detecting, locating and correcting codes where errors are affecting multiple sub-blocks. In continuation with this direction of work, we plan to work for repeated burst error affecting multiple sub-blocks. Blockwise correction of errors is very useful specially in the study of convolutional codes $[14,10]$, where the codewords consist of some smaller sub-blocks. Repeated burst error was introduced by Berardi, Dass and Verma in [1] and its general form was introduced by Dass and Verma in [5]. Repeated burst error affecting only single sub-block is studied by Dass and Madan in [7, 8] where they obtain bounds for linear code correcting and locating such errors. In this correspondence, we study the situation when repeated burst errors are occurred in multiple sub-blocks and present bounds for linear codes which can detect, locate, also correct such errors, a parallel to the work done in [3].

Now, we take the definitions of different types of errors that we are going to study in this paper. First, take the definition of a burst of length $b$, due to Fire [9], as follows.

Definition 1.1. [9] A burst of length $b$ is an n-tuple whose only nonzero components are confined to some $b$ successive positions, the first and the last of which is nonzero.

Definition 1.2. [1] An 2-repeated burst of length $b$ is an n-tuple whose only nonzero components are confined to 2 distinct sets of $b$ successive positions, the first and the last component of each set being nonzero. 
Definition 1.3. [6] An m-repeated burst of length $b$ is an $n$-tuple whose only nonzero components are confined to $m$ distinct sets of $b$ successive positions, the first and the last component of each set being nonzero.

An example of 2-repeated burst of length 4 over GF(3) is (00000010120001101000).

The number of check digits gives the information about the efficiency of a code. The efficiency of a code is inversely proportional to the number of check digits, i.e., the check digits are lesser, the code efficiency is higher.

Let us consider the number $n=f t$ and an $(n, k)$ linear code over $G F(q)$ with $f$ mutually exclusive sub-blocks of length $t$. Let $H$ be its parity check matrix and $E_{m, b, l}$ be the set of all $m$-repeated burst errors of length $b$ or less occurring in $l(\leq f)$ or less sub-blocks. Now, we state three conditions as follows.

(i) $e H^{T} \neq 0 \quad \forall e \in E_{m, b, l}$.

(ii) $e_{i} H^{T} \neq e_{j} H^{T} \forall e_{i}, e_{j} \in E_{m, b, l}$ such that $e_{i}$ and $e_{j}$ lie in the different $s(\leq l)$ sub-blocks.

(iii) $e_{i} H^{T} \neq e_{j} H^{T} \forall e_{i}, e_{j} \in E_{m, b, l}$ such that $e_{i}$ and $e_{j}$ lie in the same $s(\leq l)$ sub-blocks.

An $(n=f t, k)$ linear code capable of detecting any error from the set $E_{m, b, l}$ prevailing in at most $l(\leq f)$ corrupted sub-blocks must satisfy the condition $(i)$. We denote such type of codes by $(n=f t, k) E_{m, b, l} D$-codes. An $(n=f t, k)$ linear code for locating any error from $E_{m, b, l}$ must satisfy the conditions $(i)$ and $(i i)$. Let us denote such type of codes by $(n=f t, k)$ $E_{m, b, l} L$-codes. An $(n=f t, k)$ linear code which is capable of correcting all errors from $E_{m, b, l}$ needs to meet all the three conditions $(i)-(i i i)$ and it is denoted by an $(n=f t, k) E_{m, b, l} C$-code.

This paper is divided into four sections. Section 1 presents the introduction and preliminaries of the paper. Section 2 derives the lower and upper bounds on the number of check digits for an $(n=f t, k) E_{m, b, l} D$-code. Section 3 presents such bounds for an $(n=f t, k) E_{m, b, l} L$-code. The last section, i.e., Section 4 is devoted to obtain such bounds for an $(n=f t, k)$ $E_{m, b, l} C$-code. 


\section{Detection of repeated burst errors in multiple sub-blocks}

This section contains lower and upper bounds for detection of the errors from the set $E_{m, b, l}$.

Theorem 2.1. (Lower Bound) An $(n=f t, k) E_{m, b, l} D$-code having $r$ check digits satisfies

$$
q^{r} \geq \sum_{s=0}^{l}\left(\begin{array}{l}
l \\
s
\end{array}\right)\left(q^{m b}-1\right)^{s} .
$$

Proof. To prove this theorem, we consider the set $X$ of $n$-tuples such that components of $m$ distinct fixed sets of $b$ consecutive positions within a sub-block out of some $l$ sub-blocks are nonzero. Then, the difference of any two elements of $X$ is an error from the set $E_{m, b, l}$. Therefore, by condition $(i)$, the syndromes of the elements of $X$ must be in different cosets. Since there are

$$
\sum_{s=1}^{l}\left(\begin{array}{l}
l \\
s
\end{array}\right)\left(q^{m b}-1\right)^{s}
$$

distinct nonzero syndromes corresponding to the elements of $X$ and there are at most $q^{r}$ distinct cosets, we get

$$
q^{r} \geq \sum_{s=0}^{l}\left(\begin{array}{l}
l \\
s
\end{array}\right)\left(q^{m b}-1\right)^{s} .
$$

This proves the required result.

For 2-repeated burst error, the following corollary is obtained by taking $m=2$ in Theorem 2.1 .

Corollary 2.2. An $(n=f t, k) E_{2, b, l} D$-code having $r$ check digits satisfies

$$
q^{r} \geq \sum_{s=0}^{l}\left(\begin{array}{l}
l \\
s
\end{array}\right)\left(q^{2 b}-1\right)^{s} .
$$

Remark 2.3. Putting $m=2$ and $l=1$ in (2.1), we get

$$
q^{r} \geq q^{2 b}
$$

coinciding with Theorem 2.1 given by Berardi, Dass and Verma [1]. 
The following result is based on Varshamov- Gilbert-Sacks bound [11] and Theorem 4.17 given by Peterson and Weldon [10].

Theorem 2.4. (Upper Bound) There always exists an $(n=f t, k) E_{m, b, l} D$ code $(t>m b)$ having $r$ check digits provided

$$
\begin{aligned}
& q^{r}>q^{m(b-1)}\left\{\left(\begin{array}{c}
t-m b+(m-1) \\
m-1
\end{array}\right)(q-1)^{m-1}+\sum_{i=0}^{m-2}\left(\begin{array}{c}
t-m b+i \\
i
\end{array}\right)(q-1)^{i} q^{m-2-i}\right\} \times \\
& \sum_{s=0}^{l-1}\left(\begin{array}{c}
f-1 \\
s
\end{array}\right)\left\{q^{m(b-1)}\left[(q-1)^{m}\left(\begin{array}{c}
t-m b+m \\
m
\end{array}\right)+\sum_{i=0}^{m-1}(q-1)^{i}\left(\begin{array}{c}
t-m b+i \\
i
\end{array}\right) q^{m-1-i}\right]-1\right\}^{s} .
\end{aligned}
$$

Proof. To prove this, we will construct a suitable $(n-k) \times n$ check matrix $H$ for the code. Let us suppose that all columns of $f-1$ sub-blocks and first $j-1$ columns of the $f^{\text {th }}$ sub-block are chosen suitably and added to $H$. Now, we put down the condition to add the $j^{\text {th }}$ column $h_{j}$ such that the column $h_{j}$ should not be a linear combination of immediately preceding $b-1$ columns of the $f^{\text {th }}$ sub-block, together with $m-1$ sets of $b$ or fewer successive columns out of the first $j-b$ columns of the $f^{\text {th }}$ sub-block, along with any $m$ sets of $b$ or fewer successive columns within a sub-block chosen from any $l-1$ or less sub-blocks out of the remaining $f-1$ sub-blocks. In other words, we can write this condition as follows: 


$$
\begin{aligned}
h_{j} \neq & {\left[a_{1} h_{j-1}+a_{2} h_{j-2}+\ldots+a_{b-1} h_{j-b+1}\right] } \\
& +\left[\left(c_{i_{1}} h_{i_{1}}+c_{i_{1}+1} h_{i_{1}+1}+\ldots+c_{i_{1}-b+1} h_{i_{1}-b+1}\right)\right. \\
& +\left(c_{i_{2}} h_{i_{2}}+c_{i_{2}+1} h_{i_{2}+1}+\ldots+c_{i_{2}-b+1} h_{i_{2}-b+1}\right) \\
& \left.+\ldots+\left(c_{i_{m-1}} h_{i_{m-1}}+c_{i_{m-1}+1} h_{i_{m-1}+1}+\ldots+c_{i_{m-1}-b+1} h_{i_{m-1}-b+1}\right)\right] \\
& +\left[\left(d_{1_{1}} h_{1_{1_{1}}}+d_{1_{1_{1}}+1} h_{1_{1_{1}}+1}+\ldots+d_{1_{1_{1}}+(b-1)} h_{1_{1_{1}}+(b-1)}\right)\right. \\
& +\left(d_{1_{1_{2}}} h_{1_{1_{2}}}+d_{1_{1_{2}}+1} h_{1_{1_{2}}+1}+\ldots+d_{1_{1_{2}}+(b-1)} h_{1_{1_{2}}+(b-1)}\right)+ \\
& \left.\quad \ldots+\left(d_{1_{1_{m}}} h_{1_{1_{m}}}+d_{1_{1_{m}}+1} h_{1_{1_{m}}+1}+\ldots+d_{1_{1_{m}}+(b-1)} h_{1_{1_{m}}+(b-1)}\right)\right] \\
& + \\
& \vdots \\
+ & \\
+ & {\left[\left(d_{s_{1_{1}}} h_{s_{1_{1}}}+d_{s_{1_{1}}+1} h_{s_{1_{1}}+1}+\ldots+d_{s_{1_{1}}+(b-1)} h_{s_{1_{1}}+(b-1)}\right)\right.} \\
& +\left(d_{s_{1_{2}}} h_{s_{1_{2}}}+d_{s_{1_{2}}+1} h_{s_{1_{2}}+1}+\ldots+d_{s_{1_{2}}+(b-1)} h_{s_{1_{2}}+(b-1)}\right)+ \\
& \left.\quad \ldots+\left(d_{s_{1_{m}}} h_{s_{1_{m}}}+d_{s_{1_{m}}+1} h_{s_{1_{m}}+1}+\ldots+d_{s_{1_{m}}+(b-1)} h_{s_{1_{m}}+(b-1)}\right)\right]
\end{aligned}
$$

where $a_{i}, c_{i}, d_{w_{i_{p}}} \in G F(q), 1 \leq s \leq l-1$ and $a_{i}$ 's in the first square bracket are any immediate preceding $b-1$ columns and $c_{i}$ 's in the second square bracket are any $m-1$ sets of $b$ or less consecutive columns from the first $j-b$ columns of the $f^{\text {th }}$ sub-block, while $d_{w_{i_{p}}}$ 's are any $m$ sets of consecutive $b$ or fewer columns within a sub-block (for same value of $w$ ) chosen from any $l-1$ or fewer sub-blocks.

In the expression (2.3), the total choices of $a_{i}$ 's is $q^{b-1}$ and the choices of $c_{i}$ 's is equal to the number of $m-1$ repeated bursts of length $b$ or less in a $(j-b)$-tuple which is (see [5])

$q^{(m-1)(b-1)}\left\{\left(\begin{array}{c}j-m b+(m-1) \\ m-1\end{array}\right)(q-1)^{m-1}+\sum_{i=0}^{m-2}\left(\begin{array}{c}j-m b+i \\ i\end{array}\right)(q-1)^{i} q^{m-2-i}\right\}$.

The number of $d_{w_{i_{p}}}$ 's within a single sub-block is equivalent to the number of $m$-repeated bursts of at most length $b$ in a $t$-tuple, which is 
given as (see [5])

$$
q^{m(b-1)}\left[(q-1)^{m}\left(\begin{array}{c}
t-m b+m \\
m
\end{array}\right)+\sum_{i=0}^{m-1}(q-1)^{i}\left(\begin{array}{c}
t-m b+i \\
i
\end{array}\right) q^{m-1-i}\right]-1 .
$$

Since these errors occur in $l-1$ or less sub-blocks simultaneously, therefore the total number of linear combinations (including the zero combination) is

$$
\sum_{s=0}^{l-1}\left(\begin{array}{c}
f-1 \\
s
\end{array}\right)\left\{q^{m(b-1)}\left[(q-1)^{m}\left(\begin{array}{c}
t-m b+m \\
m
\end{array}\right)+\sum_{i=0}^{m-1}(q-1)^{i}\left(\begin{array}{c}
t-m b+i \\
i
\end{array}\right) q^{m-1-i}\right]-1\right\}^{s} .
$$

Thus, the total number of possible linear combinations of $a_{i}$ 's, $c_{i}$ 's and $d_{w_{i_{p}}}$ 's is

$$
\begin{aligned}
& q^{m(b-1)}\left\{\left(\begin{array}{c}
j-m b+(m-1) \\
m-1
\end{array}\right)(q-1)^{m-1}+\sum_{i=0}^{m-2}\left(\begin{array}{c}
j-m b+i \\
i
\end{array}\right)(q-1)^{i} q^{m-2-i}\right\} \times \\
& \sum_{s=0}^{l-1}\left(\begin{array}{c}
f-1 \\
s
\end{array}\right)\left\{q^{m(b-1)}\left[(q-1)^{m}\left(\begin{array}{c}
t-m b+m \\
m
\end{array}\right)+\sum_{i=0}^{m-1}(q-1)^{i}\left(\begin{array}{c}
t-m b+i \\
i
\end{array}\right) q^{m-1-i}\right]-1\right\}
\end{aligned}
$$

Therefore, to add the $j^{\text {th }}$ column to the $f^{\text {th }}$ sub-block, we must have

$$
\begin{aligned}
& q^{r}>q^{m(b-1)}\left\{\left(\begin{array}{c}
j-m b+(m-1) \\
m-1
\end{array}\right)(q-1)^{m-1}+\sum_{i=0}^{m-2}\left(\begin{array}{c}
j-m b+i \\
i
\end{array}\right)(q-1)^{i} q^{m-2-i}\right\} \times \\
& \sum_{s=0}^{l-1}\left(\begin{array}{c}
f-1 \\
s
\end{array}\right)\left\{q^{m(b-1)}\left[(q-1)^{m}\left(\begin{array}{c}
t-m b+m \\
m
\end{array}\right)+\sum_{i=0}^{m-1}(q-1)^{i}\left(\begin{array}{c}
t-m b+i \\
i
\end{array}\right) q^{m-1-i}\right]-1\right\}
\end{aligned}
$$

The required result is obtained by replacing $j$ by $t$ in the expression (2.5).

By putting $m=2$ in Theorem 2.4, we have the following corollary.

Corollary 2.5. There always exists an $(n=f t, k) E_{2, b, l} D$-code $(t>2 b)$ having $r$ check digits provided 


$$
\begin{aligned}
& q^{r} \quad>q^{2(b-1)}[(t-2 b+1)(q-1)+1] \times \\
& \sum_{s=0}^{l-1}\left(\begin{array}{c}
f-1 \\
s
\end{array}\right)\left\{q^{2(b-1)}\left\{(q-1)\left[\left(\begin{array}{c}
t-2 b+2 \\
2
\end{array}\right)(q-1)+\left(\begin{array}{c}
t-2 b+1 \\
1
\end{array}\right)+q\right]\right\}-1\right\} .
\end{aligned}
$$

Remark 2.6. If we put $m=2$ and $l=1$ in (2.5), we get

$$
q^{r}>q^{2(b-1)} \times[(t-2 b+1)(q-1)+1],
$$

coinciding with Theorem 2.2, Berardi, Dass and Verma [1].

Now, we provide an example of a code discussed in Theorem 2.4.

Example 2.7. Take a $(20,13)$ linear code over $G F(3)$ whose parity check matrix $H_{7 \times 20}$ is given below. Here, we take the parameters of Theorem 2.4 as $f=4, t=5, l=2, b=1, m=2$.

$$
H=\left[\begin{array}{llllllllllllllllllll}
1 & 0 & 0 & 0 & 0 & 0 & 0 & 1 & 1 & 2 & 1 & 1 & 1 & 1 & 1 & 0 & 1 & 1 & 1 & 1 \\
0 & 1 & 0 & 0 & 0 & 0 & 0 & 2 & 0 & 1 & 2 & 1 & 2 & 1 & 0 & 2 & 0 & 1 & 0 & 2 \\
0 & 0 & 1 & 0 & 0 & 0 & 0 & 1 & 1 & 2 & 2 & 2 & 2 & 1 & 1 & 0 & 2 & 0 & 2 & 1 \\
0 & 0 & 0 & 1 & 0 & 0 & 0 & 2 & 0 & 1 & 1 & 1 & 1 & 1 & 0 & 2 & 2 & 2 & 2 & 0 \\
0 & 0 & 0 & 0 & 1 & 0 & 0 & 1 & 0 & 2 & 2 & 2 & 1 & 1 & 1 & 0 & 0 & 2 & 0 & 1 \\
0 & 0 & 0 & 0 & 0 & 1 & 0 & 2 & 1 & 1 & 2 & 1 & 2 & 1 & 0 & 2 & 1 & 0 & 1 & 1 \\
0 & 0 & 0 & 0 & 0 & 0 & 1 & 1 & 0 & 0 & 1 & 1 & 2 & 1 & 1 & 0 & 1 & 2 & 2 & 2
\end{array}\right]
$$

All the 15200 syndromes of the errors from the set $E_{2,1,2}$ are found to be nonzero. This is verified by MS-EXCEL. Therefore, this code can detect 2 or less corrupted sub-blocks affected by the errors from the set $E_{2,1,2}$.

\section{Location of repeated burst errors in multiple sub-blocks}

In this section, we present both the bounds for $E_{m, b, l} L$-code.

Theorem 3.1. (Lower Bound) For an $(n=f t, k) E_{m, b, l} L$-code with $r$ number of check digits, we must have the inequality

$$
q^{r} \geq \sum_{s=0}^{l}\left(\begin{array}{l}
f \\
s
\end{array}\right)\left(q^{2 b}-1\right)^{s} .
$$


Proof. For the proof, we calculate the total number of syndromes produced by the errors from the set $E_{m, b, l}$ of which the nonzero components are confined to first $m b$ consecutive positions in each sub-block out of the $l(\leq f)$ or less corrupted sub-blocks. Then, according the conditions $(i)$ and $(i i)$, the syndromes produced by such errors from the set $E_{m, b, l}$ must be nonzero and distinct from each syndrome produced from likewise similar errors from the set $E_{m, b, l}$. There are $q^{m b}-1$ distinct nonzero such syndromes in any single sub-block and the number of corrupted sub-blocks is $l$ or less. Therefore, the total number of such nonzero syndromes is

$$
\sum_{s=1}^{l}\left(\begin{array}{l}
f \\
s
\end{array}\right)\left(q^{m b}-1\right)^{s} .
$$

As there can be at most $q^{r}$ possible distinct syndromes, including the all zero syndrome, therefore

$$
q^{r} \geq \sum_{s=0}^{l}\left(\begin{array}{l}
f \\
s
\end{array}\right)\left(q^{m b}-1\right)^{s} .
$$

By taking $m=2$ in (3.1), we have the following corollary.

Corollary 3.2. For an $(n=f t, k) E_{2, b, l} L$-code with $r$ number of check digits, we have

$$
q^{r} \geq \sum_{s=0}^{l}\left(\begin{array}{l}
f \\
s
\end{array}\right)\left(q^{2 b}-1\right)^{s} .
$$

Remark 3.3. Taking $m=2$ and $l=1$, (3.1) is reduced to

$$
q^{r} \geq 1+f\left(q^{2 b}-1\right) \text {. }
$$

This coincides with Theorem 2.1 given by Dass and Madan [8].

Theorem 3.4. (Upper Bound) An $(n=f t, k) E_{m, b, l} L$-code $(t>m b)$ having $r$ check digits, can always be constructed if

$$
\begin{aligned}
& q^{r}>q^{m(b-1)}\left\{\left(\begin{array}{c}
t-m b+(m-1) \\
m-1
\end{array}\right)(q-1)^{m-1}+\sum_{i=0}^{m-2}\left(\begin{array}{c}
t-m b+i \\
i
\end{array}\right)(q-1)^{i} q^{m-2-i}\right\} \times \\
& \sum_{s=0}^{2 l-1}\left(\begin{array}{c}
f-1 \\
s
\end{array}\right)\left\{q^{m(b-1)}\left[(q-1)^{m}\left(\begin{array}{c}
t-m b+m \\
m
\end{array}\right)+\sum_{i=0}^{m-1}(q-1)^{i}\left(\begin{array}{c}
t-m b+i \\
i
\end{array}\right) q^{m-1-i}\right]-1\right\} .
\end{aligned}
$$


Proof. To prove this theorem, we will construct a suitable $(n-k) \times n$ check matrix $H$ for the code as in Theorem 2.4. After selecting the first $f-1$ sub-blocks and first $j-1$ columns of $f^{\text {th }}$ sub-block of $H$ suitably, we go for adding the $j^{\text {th }}$ column of the $f^{\text {th }}$ sub-block to $H$ as follows.

By condition $(i)$, for detection of the corrupted sub-blocks due to errors from the set $E_{m, b, l}$, the $j^{\text {th }}$ column $h_{j}$ should not be any one of the linear combinations given by (2.3).

By condition (ii), for location of corrupted sub-blocks due to occurrence of the errors from the set $E_{m, b, l}$, the column $h_{j}$ should not a linear combination as given below:

$$
\begin{aligned}
& h_{j} \neq\left[a_{1} h_{j-1}+a_{2} h_{j-2}+\ldots+a_{b-1} h_{j-b+1}\right] \\
& +\quad\left[\left(c_{i_{1}} h_{i_{1}}+c_{i_{1}+1} h_{i_{1}+1}+\ldots+c_{i_{1}-b+1} h_{i_{1}-b+1}\right)\right. \\
& +\left(c_{i_{2}} h_{i_{2}}+c_{i_{2}+1} h_{i_{2}+1}+\ldots+c_{i_{2}-b+1} h_{i_{2}-b+1}\right) \\
& \left.+\ldots+\left(c_{i_{m-1}} h_{i_{m-1}}+c_{i_{m-1}+1} h_{i_{m-1}+1}+\ldots+c_{i_{m-1}-b+1} h_{i_{m-1}-b+1}\right)\right] \\
& +\quad\left[\left(d_{1_{1}} h_{1_{1_{1}}}+d_{1_{1_{1}}+1} h_{1_{1_{1}}+1}+\ldots+d_{1_{1_{1}}+(b-1)} h_{1_{1_{1}}+(b-1)}\right)\right. \\
& +\left(d_{1_{1_{2}}} h_{1_{1_{2}}}+d_{1_{1_{2}+1}} h_{1_{1_{2}}+1}+\ldots+d_{1_{1_{2}}+(b-1)} h_{1_{1_{2}}+(b-1)}\right)+ \\
& \left.\ldots+\left(d_{1_{1_{m}}} h_{1_{1_{m}}}+d_{1_{1_{m}}+1} h_{1_{1_{m}}+1}+\ldots+d_{1_{1_{m}}+(b-1)} h_{1_{1_{m}}+(b-1)}\right)\right] \\
& + \\
& + \\
& +\quad\left[\left(d_{s_{1}} h_{s_{1_{1}}}+d_{s_{1_{1}}+1} h_{s_{1_{1}}+1}+\ldots+d_{s_{1}+(b-1)} h_{s_{1_{1}}+(b-1)}\right)\right. \\
& +\left(d_{s_{1_{2}}} h_{s_{1_{2}}}+d_{s_{1_{2}}+1} h_{s_{1_{2}}+1}+\ldots+d_{s_{1_{2}}+(b-1)} h_{s_{1_{2}}+(b-1)}\right)+ \\
& \left.\ldots+\left(d_{s_{1_{m}}} h_{s_{1_{m}}}+d_{s_{1_{m}}+1} h_{s_{1_{m}}+1}+\ldots+d_{s_{1_{m}}+(b-1)} h_{s_{1_{m}}+(b-1)}\right)\right] \text {, }
\end{aligned}
$$

where $a_{i}, c_{i}, d_{w_{i_{p}}} \in G F(q), 1 \leq s \leq 2 l-1$ and $a_{i}$ 's in the first square bracket are any immediate preceding $b-1$ columns and $c_{i}$ 's in the second square bracket are any $m-1$ sets of $b$ or less consecutive columns from the first $j-b$ columns of the $f^{\text {th }}$ sub-block, while $d_{w_{i_{p}}}$ 's are any $m$ sets of consecutive 
$b$ or fewer columns within a sub-block (for same value of $w$ ) chosen from any $l-1$ or fewer sub-blocks.

Note that the linear combinations in (3.3) takes care of all linear combinations of (2.3). So, counting the linear combinations of (3.3) is sufficient to satisfy the conditions $(i)-(i i)$.

Therefore, the total number of possible choices of $a_{i}$ 's, $c_{i}$ 's and $d_{w_{i_{p}}}$ 's is

$$
\begin{aligned}
& q^{m(b-1)}\left\{\left(\begin{array}{c}
j-m b+(m-1) \\
m-1
\end{array}\right)(q-1)^{m-1}+\sum_{i=0}^{m-2}\left(\begin{array}{c}
j-m b+i \\
i
\end{array}\right)(q-1)^{i} q^{m-2-i}\right\} \times \\
& \sum_{s=0}^{2 l-1}\left(\begin{array}{c}
f-1 \\
s
\end{array}\right)\left\{q^{m(b-1)}\left[(q-1)^{m}\left(\begin{array}{c}
t-m b+m \\
m
\end{array}\right)+\sum_{i=0}^{m-1}(q-1)^{i}\left(\begin{array}{c}
t-m b+i \\
i
\end{array}\right) q^{m-1-i}\right]-1\right\} .
\end{aligned}
$$

This must be strictly less than $q^{r}$. Hence, we must have

$$
\begin{aligned}
& q^{r}>q^{m(b-1)}\left\{\left(\begin{array}{c}
j-m b+(m-1) \\
m-1
\end{array}\right)(q-1)^{m-1}+\sum_{i=0}^{m-2}\left(\begin{array}{c}
j-m b+i \\
i
\end{array}\right)(q-1)^{i} q^{m-2-i}\right\} \times \\
& \sum_{s=0}^{2 l-1}\left(\begin{array}{c}
f-1 \\
s
\end{array}\right)\left\{q^{m(b-1)}\left[(q-1)^{m}\left(\begin{array}{c}
t-m b+m \\
m
\end{array}\right)+\sum_{i=0}^{m-1}(q-1)^{i}\left(\begin{array}{c}
t-m b+i \\
i
\end{array}\right) q^{m-1-i}\right]-1\right\} .
\end{aligned}
$$

The required result is obtained by replacing $j$ by $t$ in the above inequality.

Corollary 3.5. An $(n=f t, k) E_{2, b, l} L$-code $(t>2 b)$ having $r$ check digits, can always be constructed if

$$
\begin{aligned}
q^{r}> & q^{2(b-1)}\{(t-2 b+1)(q-1)+1\} \sum_{s=0}^{2 l-1}\left(\begin{array}{c}
f-1 \\
s
\end{array}\right) \\
& \left\{q ^ { 2 ( b - 1 ) } \left\{\left(\begin{array}{c}
t-2 b+2 \\
2
\end{array}\right)(q-1)^{2}\right.\right. \\
& \left.\left.+\left(\begin{array}{c}
t-2 b+1 \\
1
\end{array}\right)(q-1)+q\right\}-1\right\} .
\end{aligned}
$$

Remark 3.6. Putting $m=2$ and $l=1$ in (3.2), we get

$$
\begin{aligned}
q^{r} & >q^{2(b-1)}\{(t-2 b+1)(q-1)+1\} \\
& \times\left\{1+(f-1)\left\{q^{2(b-1)}\left\{(q-1)^{2}\left(\begin{array}{c}
t-2 b+2 \\
2
\end{array}\right)+(k-1)\left(\begin{array}{c}
t-2 b+1 \\
1
\end{array}\right)+q\right\}-1\right\}\right\},
\end{aligned}
$$

which coincides with Theorem 2.3, Dass and Madan [8]. 
Example 3.7. Consider a $(20,7)$ code over $G F(3)$ with the parity check matrix $H_{13 \times 20}$ taking $f=4, t=5, l=2, b=1, m=2$ in Theorem 3.4.

$$
H=\left[\begin{array}{llllllllllllllllllll}
1 & 0 & 0 & 0 & 0 & 0 & 0 & 0 & 0 & 0 & 0 & 0 & 0 & 1 & 1 & 0 & 1 & 1 & 1 & 1 \\
0 & 1 & 0 & 0 & 0 & 0 & 0 & 0 & 0 & 0 & 0 & 0 & 0 & 1 & 0 & 2 & 0 & 1 & 0 & 2 \\
0 & 0 & 1 & 0 & 0 & 0 & 0 & 0 & 0 & 0 & 0 & 0 & 0 & 1 & 1 & 0 & 2 & 0 & 2 & 1 \\
0 & 0 & 0 & 1 & 0 & 0 & 0 & 0 & 0 & 0 & 0 & 0 & 0 & 1 & 0 & 2 & 2 & 2 & 2 & 0 \\
0 & 0 & 0 & 0 & 1 & 0 & 0 & 0 & 0 & 0 & 0 & 0 & 0 & 1 & 1 & 0 & 0 & 2 & 0 & 1 \\
0 & 0 & 0 & 0 & 0 & 1 & 0 & 0 & 0 & 0 & 0 & 0 & 0 & 1 & 0 & 2 & 1 & 0 & 1 & 1 \\
0 & 0 & 0 & 0 & 0 & 0 & 1 & 0 & 0 & 0 & 0 & 0 & 0 & 1 & 1 & 0 & 1 & 2 & 2 & 2 \\
0 & 0 & 0 & 0 & 0 & 0 & 0 & 1 & 0 & 0 & 0 & 0 & 0 & 1 & 0 & 2 & 0 & 2 & 0 & 2 \\
0 & 0 & 0 & 0 & 0 & 0 & 0 & 0 & 1 & 0 & 0 & 0 & 0 & 1 & 1 & 0 & 2 & 0 & 2 & 0 \\
0 & 0 & 0 & 0 & 0 & 0 & 0 & 0 & 0 & 1 & 0 & 0 & 0 & 1 & 0 & 2 & 1 & 1 & 1 & 0 \\
0 & 0 & 0 & 0 & 0 & 0 & 0 & 0 & 0 & 0 & 1 & 0 & 0 & 1 & 1 & 0 & 2 & 1 & 2 & 1 \\
0 & 0 & 0 & 0 & 0 & 0 & 0 & 0 & 0 & 0 & 0 & 1 & 0 & 1 & 0 & 2 & 1 & 0 & 1 & 2 \\
0 & 0 & 0 & 0 & 0 & 0 & 0 & 0 & 0 & 0 & 0 & 0 & 1 & 1 & 1 & 0 & 2 & 1 & 0 & 1 \\
& & & & & & & & & & & & & & & & &
\end{array}\right]
$$

All the 15200 syndromes of the errors from the set $E_{2,1,2}$ are nonzero and distinct in different set of $l$ or less sub-blocks (verified with the help of $M S$-EXCEL). Therefore, this code is an example of $E_{2,1,2} L$-code.

\section{Correction of repeated burst errors occurring in multiple sub-blocks}

In this section, the study is about the codes capable of correcting the errors from the set $E_{m, b, l}$.

Theorem 4.1. (Lower Bound) An $(n=f t, k) E_{m, b, l} C$-code $(t>m b)$ with $r$ parity check digits must satisfy

$q^{r} \geq \sum_{s=0}^{l}\left(\begin{array}{l}f \\ s\end{array}\right)\left\{q^{m(b-1)}\left[(q-1)^{m}\left(\begin{array}{c}t-m b+m \\ m\end{array}\right)+\sum_{i=0}^{m-1}(q-1)^{i}\left(\begin{array}{c}t-m b+i \\ i\end{array}\right) q^{m-1-i}\right]-1\right\}^{s}$.

Proof. In this case also, we prove the result by counting the total number of syndromes satisfying conditions $(i)-($ iii $)$. We count all possible number of errors in the set $E_{m, b, l}$. The number of $m$-repeated burst errors of length $b$ or less occurred in one sub-block of length $t$ (excluding zero 
vector) is given by (see [5])

$$
q^{m(b-1)}\left[(q-1)^{m}\left(\begin{array}{c}
t-m b+m \\
m
\end{array}\right)+\sum_{i=0}^{m-1}(q-1)^{i}\left(\begin{array}{c}
t-m b+i \\
i
\end{array}\right) q^{m-1-i}\right]-1 .
$$

Since the number of corrupted sub-blocks is $l(\leq f)$ or less, the total number of errors from the set $E_{m, b, l}$, excluding the zero vector, is

$\sum_{s=1}^{l}\left(\begin{array}{l}f \\ s\end{array}\right)\left\{q^{m(b-1)}\left[(q-1)^{m}\left(\begin{array}{c}t-m b+m \\ m\end{array}\right)+\sum_{i=0}^{m-1}(q-1)^{i}\left(\begin{array}{c}t-m b+i \\ i\end{array}\right) q^{m-1-i}\right]-1\right\}^{s}$.

Therefore, total number of nonzero and distinct syndromes (according to conditions $(i)-(i i i))$ is given by (4.1). Since there are at most $q^{r}$ syndromes, we get

$q^{r} \geq \sum_{s=0}^{l}\left(\begin{array}{l}f \\ s\end{array}\right)\left\{q^{m(b-1)}\left[(q-1)^{m}\left(\begin{array}{c}t-m b+m \\ m\end{array}\right)+\sum_{i=0}^{m-1}(q-1)^{i}\left(\begin{array}{c}t-m b+i \\ i\end{array}\right) q^{m-1-i}\right]-1\right\}^{s}$.

Corollary 4.2. An $(n=f t, k) E_{2, b, l} C$-code $(t>2 b)$ with $r$ parity check digits must satisfy

$q^{r} \geq \sum_{s=0}^{l}\left(\begin{array}{l}f \\ s\end{array}\right)\left\{q^{2(b-1)}\left\{\left(\begin{array}{c}t-2 b+2 \\ 2\end{array}\right)(q-1)^{2}+\left(\begin{array}{c}t-2 b+1 \\ 1\end{array}\right)(q-1)+q\right\}-1\right\}^{s}$.

Remark 4.3. By taking $m=2$ and $l=1$, the result obtained in (4.2) reduces to

$q^{r} \geq 1+f\left\{q^{2(b-1)}\left\{\left(\begin{array}{c}t-2 b+2 \\ 2\end{array}\right)(q-1)^{2}+\left(\begin{array}{c}t-2 b+1 \\ 1\end{array}\right)(q-1)+q\right\}-1\right\}$.

This coincides with Theorem 1, Dass and Madan [7].

Theorem 4.4. (Upper Bound) The existence of an $(n=f t, k) E_{m, b, l} C$ code $(t>2 m b)$ is always ensured provided 


$$
\begin{aligned}
q^{r}> & \left.q^{2 m(b-1)}\left[\begin{array}{c}
t-2 m b+2 m-1 \\
2 m-1
\end{array}\right)(q-1)^{2 m-1}+\sum_{i=0}^{2 m-2}(q-1)^{i}\left(\begin{array}{c}
t-2 m b+i \\
i
\end{array}\right) q^{2 m-2-i}\right] \times \\
& {\left[\sum _ { s = 0 } ^ { l - 1 } ( \begin{array} { c } 
{ f - 1 } \\
{ s }
\end{array} ) \left\{q ^ { 2 m ( b - 1 ) } \left\{(q-1)^{2 m(b-1)}\left(\begin{array}{c}
t-2 m b+2 m \\
2 m
\end{array}\right)\right.\right.\right.} \\
& \left.\left.\left.\left.+\sum_{i=0}^{2 m-1}(q-1)^{i} \underset{i}{(-2 m b+i}\right) q^{2 m-1-i}\right\}-1\right\}\right] \\
& +q^{m(b-1)}\left\{\left(\begin{array}{c}
t-m b+(m-1) \\
m-1
\end{array}\right)(q-1)^{m-1}+\sum_{i=0}^{m-2}\left(\begin{array}{c}
t-m b+i \\
i
\end{array}\right)(q-1)^{i} q^{m-2-i}\right\} \times \\
& \sum_{s=l}^{2 l-1}\left(\begin{array}{c}
f-1 \\
s
\end{array}\right)\left\{q ^ { m ( b - 1 ) } \left[(q-1)^{m}\left(\begin{array}{c}
t-m b+m \\
m
\end{array}\right)\right.\right. \\
& \left.\left.+\sum_{i=0}^{m-1}(q-1)^{i}\left(\begin{array}{c}
t-m b+i \\
i
\end{array}\right) q^{m-1-i}\right]-1\right\},
\end{aligned}
$$

where $r$ is the number of check digits of the code.

Proof. In line with the proof of Theorem 2.4 and Theorem 3.4, let us assume that the first $f-1$ sub-blocks and the first $j-1$ columns of the $f^{t h}$ sub-block of the parity check matrix $H_{(n-k) \times n}$ of the desired code are chosen suitably. Now for addition of the $j^{\text {th }}$ column $h_{j}$ of $f^{\text {th }}$ sub-block to the matrix $H$, by conditions $(i)-(i i i)$, the syndromes produced by the errors from the set $E_{m, b, l}$ must be nonzero and distinct from the syndromes produced by other any $E_{m, b, l}$ errors from not only in the same set, but also in other set of $l$ or fewer sub-blocks out of $f$ sub-blocks.

According to conditions $(i)-(i i)$, the linear combinations that $h_{j}$ can not be equal to is given by (3.3).

According to condition (iii), in order to put the column $h_{j}$ as $j^{\text {th }}$ column, the following condition needs to be satisfied. 


$$
\begin{aligned}
& h_{j} \neq \quad\left[a_{1} h_{j-1}+a_{2} h_{j-2}+\ldots+a_{b-1} h_{j-b+1}\right] \\
& +\left(c_{i_{1}} h_{i_{1}}+c_{i_{1}+1} h_{i_{1}+1}+\ldots+c_{i_{1}-b+1} h_{i_{1}-b+1}\right) \\
& +\left(c_{i_{2}} h_{i_{2}}+c_{i_{2}+1} h_{i_{2}+1}+\ldots+c_{i_{2}-b+1} h_{i_{2}-b+1}\right) \\
& \left.+\ldots+\left(c_{i_{2 m-1}} h_{i_{2 m-1}}+c_{i_{2 m-1}+1} h_{i_{2 m-1}+1}+\ldots+c_{i_{2 m-1}-b+1} h_{i_{2 m-1}-b+1}\right)\right] \\
& +\left[\left(d_{1_{1}} h_{1_{1_{1}}}+d_{1_{1_{1}}+1} h_{1_{1_{1}+1}}+\ldots+d_{1_{1_{1}}+(b-1)} h_{\left.1_{1_{1}+(b-1)}\right)}\right)\right. \\
& +\left(d_{1_{1_{2}}} h_{1_{1_{2}}}+d_{1_{1_{2}+1}} h_{1_{1_{2}+1}}+\ldots+d_{1_{1_{2}}+(b-1)} h_{1_{1_{2}}+(b-1)}\right)+ \\
& \left.\ldots+\left(d_{1_{1_{2 m}}} h_{1_{1_{2 m}}}+d_{1_{1_{2 m}}+1} h_{1_{1_{2 m}}+1}+\ldots+d_{1_{1_{2 m}}+(b-1)} h_{1_{1_{2 m}}+(b-1)}\right)\right] \\
& + \\
& \vdots \\
& + \\
& +\left[\left(d_{s_{1}} h_{s_{1}}+d_{s_{1}+1} h_{s} 1_{1}+1+\ldots+d_{s_{1}+(b-1)} h_{s_{1_{1}}+(b-1)}\right)\right. \\
& +\left(d_{s_{2}} h_{s_{1}}+d_{s_{1}+1} h_{s_{1_{2}}+1}+\ldots+d_{s 1_{2}+(b-1)} h_{s 1_{2}+(b-1)}\right)+ \\
& \left.\ldots+\left(d_{s_{12 m}} h_{s_{1_{2 m}}}+d_{s_{1_{2 m}}+1} h_{s_{1_{2 m}}+1}+\ldots+d_{s_{1_{2 m}}+(b-1)} h_{s_{1_{2 m}}+(b-1)}\right)\right] \text {, }
\end{aligned}
$$

where $a_{i}, c_{i}, d_{w_{i_{p}}} \in G F(q), 1 \leq s \leq l-1$ and $a_{i}$ 's in the first square bracket are any immediate preceding $b-1$ columns and $c_{i}$ 's in the second square bracket are any $2 m-1$ sets of $b$ or less consecutive columns from the first $j-b$ columns of the $f^{\text {th }}$ sub-block, while $d_{w_{i_{p}}}$ 's are any $2 m$ sets of consecutive $b$ or fewer columns within a sub-block (for same value of $w$ ) chosen from any $l-1$ or fewer sub-blocks.

In this expression, the total choices of $a_{i}$ 's are $q^{b-1}$ and the number of choices of $c_{i}$ has the same as the number of $(2 m-1)$-repeated burst of length at most $b$ in a $(j-b)$-tuple. Therefore, the total number of choices of $a_{i}$ and $c_{i}$ is given by (refer Theorem 2.2, [5])

$$
q^{2 m(b-1)}\left\{\left(\begin{array}{c}
j-2 m b+2 m-1 \\
2 m-1
\end{array}\right)(q-1)^{2 m-1}+\sum_{i=0}^{2 m-2}(q-1)^{i}\left(\begin{array}{c}
j-2 m b+i \\
i
\end{array}\right) q^{2 m-2-i}\right\} .
$$


The total choices of the coefficients $d_{w_{i_{p}}}$ 's in the expression (4.4) has the same number as that of $2 m$-repeated bursts of length at most $b$ in any $l-1$ or fewer sub-blocks (each of length $t$ ). So, the total choices of $d_{w_{i_{p}}}$ 's is

$$
\begin{aligned}
& \sum_{s=0}^{l-1}\left(\begin{array}{c}
f-1 \\
s
\end{array}\right)\left\{q ^ { 2 m ( b - 1 ) } \left\{(q-1)^{2 m(b-1)}\left(\begin{array}{c}
t-2 m b+2 m \\
2 m
\end{array}\right)\right.\right. \\
& \left.\left.+\sum_{i=0}^{2 m-1}(q-1)^{i}\left(\begin{array}{c}
t-2 m b+i \\
i
\end{array}\right) q^{2 m-1-i}\right\}-1\right\}^{s} .
\end{aligned}
$$

Thus, the total number of linear combinations in expression (4.4) is given by

$$
\text { Expr.(4.5) × Expr.(4.6) }
$$

i.e.

$$
\begin{aligned}
q^{2 m(b-1)} & {\left.\left[\begin{array}{c}
j-2 m b+2 m-1 \\
2 m-1
\end{array}\right)(q-1)^{2 m-1}+\sum_{i=0}^{2 m-2}(q-1)^{i}\left(\begin{array}{c}
j-2 m b+i \\
i
\end{array}\right) q^{2 m-2-i}\right] \times } \\
& {\left[\sum _ { s = 0 } ^ { l - 1 } ( \begin{array} { c } 
{ f - 1 } \\
{ s }
\end{array} ) \left\{q ^ { 2 m ( b - 1 ) } \left\{(q-1)^{2 m(b-1)}\left(\begin{array}{c}
t-2 m b+2 m \\
2 m
\end{array}\right)\right.\right.\right.} \\
& \left.\left.\left.+\sum_{i=0}^{2 m-1}(q-1)^{i}(\underset{i}{t-2 m b+i}) q^{2 m-1-i}\right\}-1\right\}\right] .
\end{aligned}
$$

Further, there are some linear combinations on R.H.S. of (3.3) which are not included on R.H.S. of (4.4) and this number is

$$
\begin{aligned}
& q^{m(b-1)}\left\{\left(\begin{array}{c}
t-m b+(m-1) \\
m-1
\end{array}\right)(q-1)^{m-1}+\sum_{i=0}^{m-2}\left(\begin{array}{c}
t-m b+i \\
i
\end{array}\right)(q-1)^{i} q^{m-2-i}\right\} \times \\
& \sum_{s=l}^{2 l-1}\left(\begin{array}{c}
f-1 \\
s
\end{array}\right)\left\{q ^ { m ( b - 1 ) } \left[(q-1)^{m}\left(\begin{array}{c}
t-m b+m \\
m
\end{array}\right)\right.\right. \\
&\left.\left.+\sum_{i=0}^{m-1}(q-1)^{i}\left(\begin{array}{c}
t-m b+i \\
i
\end{array}\right) q^{m-1-i}\right]-1\right\}^{s} .
\end{aligned}
$$

Therefore, the total number of linear combinations satisfying conditions (i) $-($ iii $)$ is

$$
\text { Expr.(4.7) +Expr.(4.8). }
$$


Thus, we have

$$
\begin{aligned}
q^{r}> & \left.q^{2 m(b-1)}\left[\begin{array}{c}
j-2 m b+2 m-1 \\
2 m-1
\end{array}\right)(q-1)^{2 m-1}+\sum_{i=0}^{2 m-2}(q-1)^{i}\left(\begin{array}{c}
j-2 m b+i \\
i
\end{array}\right) q^{2 m-2-i}\right] \times \\
& {\left[\sum _ { s = 0 } ^ { l - 1 } ( \begin{array} { c } 
{ f - 1 } \\
{ s }
\end{array} ) \left\{q ^ { 2 m ( b - 1 ) } \left\{(q-1)^{2 m(b-1)}\left(\begin{array}{c}
t-2 m b+2 m \\
2 m
\end{array}\right)\right.\right.\right.} \\
& \left.\left.\left.+\sum_{i=0}^{2 m-1}(q-1)^{i}\left(\begin{array}{c}
t-2 m b+i \\
i
\end{array}\right) q^{2 m-1-i}\right\}-1\right\}\right] \\
& +q^{m(b-1)}\left\{\left(\begin{array}{c}
t-m b+(m-1) \\
m-1
\end{array}\right)(q-1)^{m-1}+\sum_{i=0}^{m-2}\left(\begin{array}{c}
t-m b+i \\
i
\end{array}\right)(q-1)^{i} q^{m-2-i}\right\} \times \\
& \sum_{s=l}^{2 l-1}\left(\begin{array}{c}
f-1 \\
s
\end{array}\right)\left\{q ^ { m ( b - 1 ) } \left[(q-1)^{m}\left(\begin{array}{c}
t-m b+m \\
m
\end{array}\right)\right.\right. \\
& \left.\left.+\sum_{i=0}^{m-1}(q-1)^{i}\left(\begin{array}{c}
t-m b+i \\
i
\end{array}\right) q^{m-1-i}\right]-1\right\} .
\end{aligned}
$$

Replacing $j$ by $t$ in (4.9) gives the required result.

Corollary 4.5. (Upper Bound) There always exists an $(n=f t, k) E_{2, b, l} C$ code with $(t>4 b)$ having $r$ parity check digits provided that

$$
\begin{aligned}
q^{r}> & {\left[q^{4(b-1)}\left[\left(\begin{array}{c}
t-4 b+3 \\
3
\end{array}\right)(q-1)^{3}+\sum_{i=0}^{2}(q-1)^{i}\left(\begin{array}{c}
t-4 b+i \\
i
\end{array}\right) q^{4-2-i}\right\}\right.} \\
& \times\left\{\sum _ { s = 0 } ^ { l - 1 } ( \begin{array} { c } 
{ f - 1 } \\
{ s }
\end{array} ) \left\{q ^ { 4 ( b - 1 ) } \left\{\left(\begin{array}{c}
t-4 b+4 \\
4
\end{array}\right)(q-1)^{4}+\right.\right.\right. \\
& \left.\left.\left.\sum_{i=0}^{3}(q-1)^{i}\left(\begin{array}{c}
t-4 b+i \\
i
\end{array}\right) q^{4-1-i}\right\}-1\right\}\right] \\
& +\left[q ^ { 2 ( b - 1 ) } \{ ( t - 2 b + 1 ) ( q - 1 ) + 1 \} \left\{\sum_{s=l}^{2 l-1}\left(\begin{array}{c}
f-1 \\
s
\end{array}\right) \times\right.\right. \\
& {\left.\left.\left[q^{2(b-1)}\left\{\left(\begin{array}{c}
t-2 b+2 \\
2
\end{array}\right)(q-1)^{2}+\left(\begin{array}{c}
t-2 b+1 \\
1
\end{array}\right)(q-1)+q\right\}-1\right]^{s}\right\}\right] . }
\end{aligned}
$$

Remark 4.6. Take $m=2$ and $l=1$, the result (4.3) reduces to 


$$
\begin{aligned}
q^{r}> & q^{4(b-1)}\left\{\left(\begin{array}{c}
t-4 b+3 \\
3
\end{array}\right)(q-1)^{3}+\sum_{i=0}^{2}(q-1)^{i}\left(\begin{array}{c}
t-4 b+i \\
i
\end{array}\right) q^{2-i}\right\} \\
& +q^{2(b-1)}\{(t-2 b+1)(q-1)+1\} \times \\
& (f-1)\left[q^{2(b-1)}\left\{\left(\begin{array}{c}
t-2 b+2 \\
2
\end{array}\right)(q-1)^{2}+\left(\begin{array}{c}
t-2 b+1 \\
1
\end{array}\right)(q-1)+q\right\}-1\right],
\end{aligned}
$$

coinciding with Theorem 2, Dass and Madan [7].

Now, an example of an $E_{2, b, l} C$ code is provided below.

Example 4.7. Consider a $(15,4)$ linear code over $G F(3)$ with the parity check matrix $H_{11 \times 15}$ for Theorem 4.4 by choosing $f=3, t=5, l=2$, $b=1, m=2$.

$$
H=\left[\begin{array}{lllllllllllllll}
1 & 0 & 0 & 0 & 0 & 0 & 0 & 0 & 0 & 0 & 0 & 1 & 1 & 1 & 1 \\
0 & 1 & 0 & 0 & 0 & 0 & 0 & 0 & 0 & 0 & 0 & 1 & 0 & 1 & 0 \\
0 & 0 & 1 & 0 & 0 & 0 & 0 & 0 & 0 & 0 & 0 & 1 & 1 & 0 & 2 \\
0 & 0 & 0 & 1 & 0 & 0 & 0 & 0 & 0 & 0 & 0 & 1 & 2 & 2 & 2 \\
0 & 0 & 0 & 0 & 1 & 0 & 0 & 0 & 0 & 0 & 0 & 1 & 0 & 2 & 0 \\
0 & 0 & 0 & 0 & 0 & 1 & 0 & 0 & 0 & 0 & 0 & 1 & 1 & 0 & 1 \\
0 & 0 & 0 & 0 & 0 & 0 & 1 & 0 & 0 & 0 & 0 & 1 & 0 & 1 & 1 \\
0 & 0 & 0 & 0 & 0 & 0 & 0 & 1 & 0 & 0 & 0 & 1 & 1 & 1 & 0 \\
0 & 0 & 0 & 0 & 0 & 0 & 0 & 0 & 1 & 0 & 0 & 1 & 2 & 0 & 2 \\
0 & 0 & 0 & 0 & 0 & 0 & 0 & 0 & 0 & 1 & 0 & 1 & 0 & 2 & 1 \\
0 & 0 & 0 & 0 & 0 & 0 & 0 & 0 & 0 & 0 & 1 & 1 & 1 & 2 & 2
\end{array}\right]
$$

This matrix is constructed by the synthesis procedure discussed in Theorem 4.4. All the 7650 syndromes of the errors from the set $E_{2,1,2}$ are nonzero and distinct altogether (verified by MS-EXCEL). Therefore, this $E_{2,1,2} C$-code code can correct 2 or less corrupted sub-blocks affected by errors from the set $E_{2,1,2}$.

\section{Acknowledgement}

The authors are very thankful to the respected anonymous reviewer(s) for the meticulous reading and valuable suggestions which improves the quality of this paper. 


\section{References}

[1] L. Berardi, B. K. Dass, and R. Verma, "On 2-repeated burst error detecting Linear Codes", Journal of statistical theory and practice, vol. 3, no. 2, pp. 381-391, Jun.2009, doi: 10.1080/ 15598608.2009.10411931

[2] P. K. Das, "Location of multiple sub-blocks with burst errors", Electronic notes in discrete mathematics, vol. 63, pp. 117-123, Dec. 2017, doi: 10.1016/j.endm.2017.11.006

[3] P. K. Das, "Codes detecting, locating and correcting random errors occurring in multiple sub-blocks", Proyecciones (Antofagasta. En línea), vol. 38, no. 1, pp. 129-144, Mar. 2019, doi: 10.4067/ S071609172019000100129

[4] P. K. Das and L. K. Vashisht, "Error locating codes by using blockwisetensor product of blockwise detecting/correcting codes", Khayyam journal of mathematics, vol. 2, no. 1, pp. 6-17, 2016, doi: 10.22034/ KJM.2016.14572

[5] B. K. Dass and R. Verma, "Repeated burst error correcting linear codes", Asian-european journal of mathematics, vol. 1, no. 3, pp. 303-335, 2008, doi: 10.1142/ S1793557108000278

[6] B. K. Dass and R. Verma, "Repeated burst error detecting linear codes", Ratio mathematica, vol. 19, pp. 25-30, 2009. [On line]. Available: https:/ / bit.ly/ 3eosbVj

[7] B. K. Dass and S. Madan, "Blockwise repeated burst error correcting linear codes", Ratio mathematica, vol. 20, pp. 97-126, 2010. [On line]. Available: https:/ / bit.ly/ 3eqLqxf

[8] B. K. Dass and S. Madan, "Repeated burst error locating linear codes", Discrete mathematics, algorithms and applications, vol. 2, no. 2, pp. 181-188, 2010, doi: 10.1142/ S1793830910000553

[9] P. Fire, "A class of multiple-error-correction binary codes for nonindependent errors", Thesis Engineer, Stanford University, Dept. of Electrical Engineering, 1959.

[10] W. W. Peterson and E. J. Weldon, Error correcting codes, 2nd ed. Cambridge, MA: MTT Press, 1972.

[11] G. E. Sacks, "Multiple error correction by means of parity-checks", IRE transactions on information theory, vol. 4, no. 4, pp. 145-147, Dec. 1958, doi: 10.1109/ IRETIT.1958.6741947 
[12] J. K. Wolf, "On an extended class of error-locating codes”, Information and control, vol. 8, no. 2, pp. 163-169, Apr.1965, doi: 10.1016/ S0019-9958(65)90066-5

[13] J. K. Wolf and B. Elspas, "Error-locating codes--A new concept in error control", IEEE transactions on information theory, vol. 9, no. 2, pp. 113-117, Apr.1963, doi: 10.1109/ TIT.1963.1057813

[14] J. M. Wozencraft, "Sequential decoding for reliable communication", IRE National Convention Record, vol. 5, no. 2, pp. 11-25, 1957. 